\title{
Comparison of the EZ-Cap Recapper with the Mayo Recapper for the Prevention of Needlesticks
}

\author{
Chad A. Dewhirst and Joseph C. Hung \\ Division of Nuclear Medicine, Department of Radiology, Mayo Clinic, Rochester, Minnesota
}

\begin{abstract}
The purpose of this project was the development of a device that improves the design of our current capping block, the Mayo recapper. The major challenges for design and improvement included creating a device that is simple to use and can be applied throughout our department. We wanted a recapper device that increased safety and minimized the potential for needlesticks. Simplicity was another important factor, along with versatility and low cost. A new recapper, called EZ-Cap, was developed, and a comparison study was conducted to evaluate the pros and cons of the EZ-Cap recapper and the Mayo recapper. Methods: Nuclear medicine technologists $(n=10)$ in our department used each device when administering patient injections. At the conclusion of their patient injection rotation, they recorded on a survey sheet the pros and cons of each device. The results of this survey were used to evaluate the effectiveness, comfort level during use, and safety of each recapping device. We used a 2-level scoring system to help determine which device was more favorable. The first level focused on comfort and convenience and was given a score of +1 or -1 . The second level focused on safety and was given a score of +2 or -2 . Because we believed that safety was a high priority for our capping blocks, this level received a higher score than the first level. Results: The Mayo recapper was the device preferred by 9 of 10 technologists surveyed. The EZ-Cap recapper had several technical issues that made it difficult to use and that could potentially lead to safety concerns. According to our scoring system, the Mayo recapper received a score of +9 for its pros and -4 for its cons. By comparison, the EZ-Cap recapper received a score of +7 for its pros and -16 for its cons. Conclusion: Our results show that the Mayo recapper was the device of choice because its pros outweighed its cons. However, we will continually improve the effectiveness of the Mayo recapper to prevent needlesticks.
\end{abstract}

Key Words: capping block; needles; needlestick; recapper; syringes

J Nucl Med Technol 2008; 36:151-154

DOI: 10.2967/jnmt.108.051938

$\mathbf{N}$ eedlesticks are a major concern for health care professionals. Studies have shown that approximately 562-839

Received Feb. 19, 2008; revision accepted Jul. 30, 2008.

For correspondence or reprints contact: Joseph C. Hung, Department of Radiology, Mayo Clinic, 200 First St. SW, Rochester, MN 55905.

E-mail: jhung@mayo.edu

COPYRIGHT @ 2008 by the Society of Nuclear Medicine, Inc. needlestick injuries occur per 1,000 health care employees per year (1). These needlesticks may lead to contraction of any of more than 23 infectious diseases that can be transmitted by needlestick (2). Any health care professional who is required to access a patient's blood is at risk of a needlestick or a disease transmitted through contamination.

A simple recapping device would help health care workers with safety compliance and prevention of needlesticks. Surveys have found that health care workers continue the practice of recapping needles, do not wear appropriate protective equipment, and dispose of needles improperly, even though universal precautions and work practice controls are in place at hospitals (1). Many hospitals have found that the price of a new recapping device and the training of staff to use it limit the ability to purchase such a device (1); thus, the availability of an inexpensive device would be advantageous.

Karen Daley, a former president of both the Massachusetts Nurses Association and the Massachusetts Center for Nursing, has found that $85 \%$ of needlestick exposures can be prevented with safer recapper devices (3). Daley also had a key role in the passage of the 2000 Needlestick Safety and Prevention Act (3). This legislation requires health care facilities to provide safety-engineered devices for needles and sharps for employees who deliver direct care to patients (3).

Since 1999, employees in our nuclear medicine division have been involved in 10 needlestick incidents. These incidents differed in type and in the equipment involved (Table 1).

Previously, the Mayo recapper (Fig. 1) was used as our primary capping block. This device was designed and produced at Mayo Clinic. However, we wanted to improve our capping-block design so that the recapper could be operated (capping or uncapping) with a single hand, could be used with both butterfly needles and syringes, and could be used on many different surfaces. A new recapper device was developed and was called EZ-Cap (Fig. 2). This device was manufactured by a former employee of Mayo Clinic and addressed some of the deficiencies found in the Mayo recapper.

The purpose of our study was to determine whether the EZ-Cap recapper was an improvement over the Mayo recapper and to evaluate the pros and cons of each device. 
TABLE 1

The 10 Needlestick Injuries Occurring from January 1, 1999, to May 31, 2008, in the Division of Nuclear Medicine at Mayo Clinic

\begin{tabular}{cc}
\hline \multicolumn{1}{c}{ Incident } & $n$ \\
\hline Involving a syringe needle & 6 \\
When uncapping a syringe & 2 \\
When recapping a syringe & 3 \\
When removing a needle from a patient & 1 \\
Involving a butterfly needle & 4 \\
When uncapping a butterfly needle & 0 \\
When recapping a butterfly needle & 2 \\
When removing a needle from a patient & 2 \\
\hline
\end{tabular}

\section{MATERIALS AND METHODS}

The Mayo recapper consists of a cylindric white plastic base with 2 holes in its top (Fig. 1). One hole is used for a needle cap; the other, for a butterfly needle. To use this device to recap a needle, the nuclear medicine technologist simply inverts the syringe and presses it into the recapper. However, to uncap a needle, the technologist must use both hands to pull the cap off manually. This action of uncapping a needle is a major drawback that may increase the risk of a needlestick injury. When removed, the needle cap can be placed in the designated hole. Our technologists have been using this device for about $8 \mathrm{y}$. This device is simple in its design and is straightforward in its use.

The EZ-Cap recapper consists of a slanted needle holder connected to a suction cup (Fig. 2) that can adhere to any flat surface. To use this device, the technologist pushes the needle cap into the holder and twists the syringe to remove the cap. The needle is recapped by pushing back into the holder and twisting in the opposite direction until the cap is secure on the syringe. These

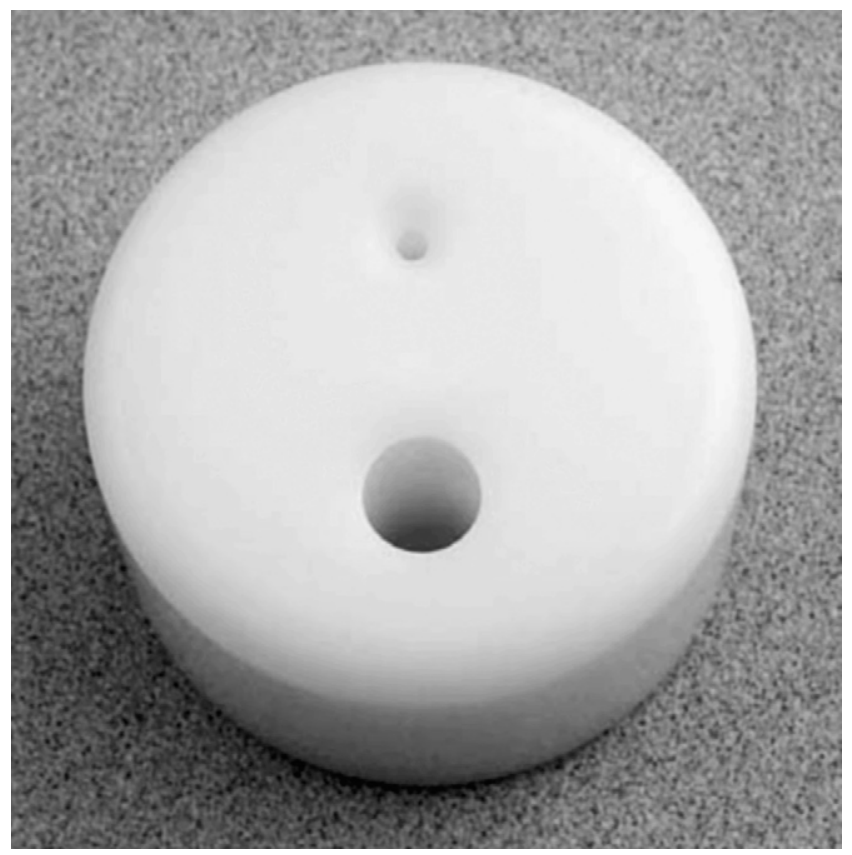

FIGURE 1. Mayo recapper. Large hole can house regular needle cap; small hole, butterfly needle.

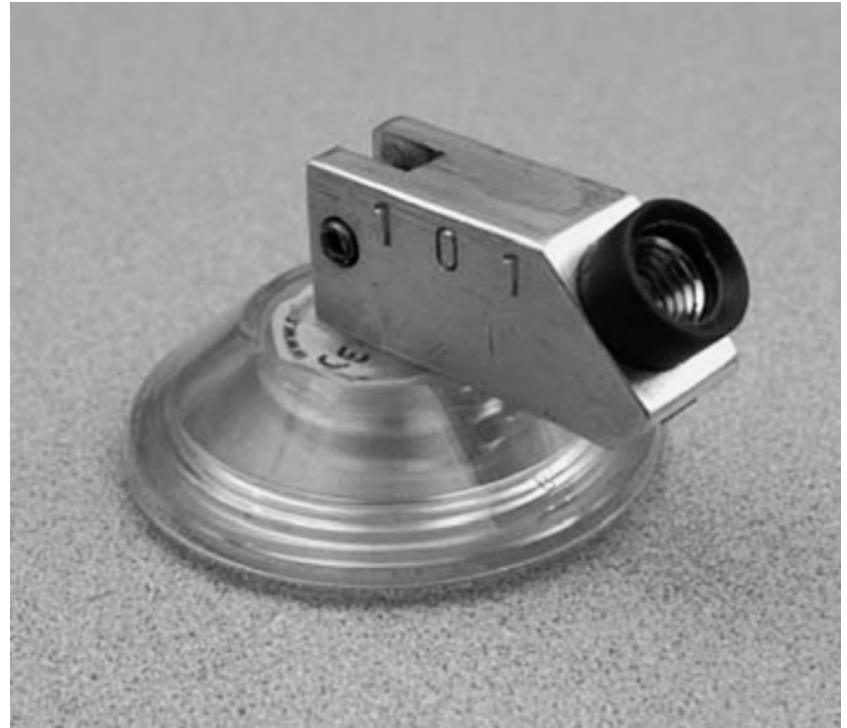

FIGURE 2. EZ-Cap recapper.

techniques allow capping and recapping operations with 1 hand. This device has been used at our institution for only about $7 \mathrm{y}$.

Ultimately, we wanted a capping block that retained the pros of the Mayo recapper but improved on its cons. We wanted a device that limited the potential for needlesticks and was simple to use, versatile, and inexpensive. This project helped determine whether we had achieved these goals with the EZ-Cap recapper.

The technologists surveyed for this study had a range of experience. They included student interns with a few months' experience to technologists with more than $10 \mathrm{y}$ of experience in nuclear medicine. Any student working in our department is required to work with an experienced staff technologist.

A survey sheet was used to gather the technologists' views about the pros and cons of the Mayo recapper and the EZ-Cap recapper. The survey sheet was divided into 2 columns. One column listed a pros section and the opposite column listed a cons section for each recapping device. There were no questions to answer, but the survey sheet allowed our technologists to describe what they believed were the most important factors of each capping block. The written comments were grouped together, and the most important factors for each device are discussed herein.

The survey responses were reviewed and graded according to a 2-level scoring system. The first level focused primarily on comfort and convenience. A score of +1 or -1 was assigned to responses in this level. For example, a statement about the device's aiding the technologist in comfort or convenience was given a +1 score. A statement that the recapper hindered the technologist's comfort or convenience was given a -1 . The second level focused on safety, for which a score of +2 or -2 was given. The grading was similar to the first level: a positive score was assigned to statements about the recapper's aiding in safety, and a negative score was assigned to statements about its hindering safety. This second level received a higher score because we believed that safety was a major concern when needlestick injuries were possible. Finally, the scores were combined for each recapping device, to achieve a final number (positive or negative) for each device. This cumulative score allowed us to make an unbiased decision and to determine which device our technologists preferred. 


\section{RESULTS}

A total of 10 nuclear medicine technologists participated in our evaluation project during their patient injection rotation. At the conclusion of the rotation, each technologist completed the survey sheet.

\section{Pros of the Mayo Recapper}

The Mayo recapper has holes for 2 different needle caps, a feature stated by 4 of the 10 technologists surveyed. Also, 4 of the technologists surveyed stated that the absence of a suction cup on the device allows for easy movement, and no twisting is required to remove the needle cap. The Mayo recapper is equipped with 5 small, nonslip feet to aid in keeping the device stationary. It is simple to use, and the user can easily see when the needle is recapped, features stated by 4 technologists. Also, the Mayo recapper is the device that most nuclear medicine technologists felt comfortable using.

\section{Cons of the Mayo Recapper}

The Mayo recapper is not a hands-free device, and a level surface is required for its use, characteristics noted by 6 of the 10 technologists surveyed. Additionally, 1 technologist stated that the angle used to recap a syringe is not ideal, compared with the angle of the EZ-Cap recapper. Finally, 1 technologist noted that the Mayo recapper is not optimal for uncapping a syringe when compared with the EZ-Cap recapper.

\section{Pros of the EZ-Cap Recapper}

The reported pros of the EZ-Cap recapper (Fig. 2) included its design for single-handed use, with the angle of the needle holder allowing faster and easier recapping. This observation was stated by 8 of the 10 technologists surveyed. Three technologists commented that the slanted angle of the device is much more favorable for recapping than the angle of the Mayo recapper. Finally, the suction cup allows the EZ-Cap recapper to be stable and to adhere to many different surfaces.

\section{Cons of the EZ-Cap Recapper}

The EZ-Cap recapper is difficult to use when one is twisting the needle cap off, and sometimes, the entire needle is accidentally pulled off. This difficulty was reported by 5 of the 10 technologists surveyed. Also, when the syringe is pushed into the needle holder, there is a potential to push on the plunger of the syringe. Furthermore, the suction cup of the device sometimes hinders its movement. The EZ-Cap recapper has room for only 1 needle cap at a time, and butterfly needles cannot be used with this recapping device. Additionally, technologists need to manipulate an exposed needle to cap or uncap a syringe and are required to twist their wrist and the syringe to cap or uncap a syringe. Four technologists reported that this action makes the use of this device difficult and awkward. We also concluded that the EZ-Cap recapper would be a more expensive capping block because of its complicated nature and its many components, compared with the Mayo recapper.

\section{Final Scores}

These pros and cons were graded with the scoring system. Results for the Mayo recapper were +9 for the pros and -4 for the cons. The EZ-Cap recapper received scores of +7 for the pros and -16 for the cons. The final, combined result for the Mayo recapper was +5 ; the final, combined score for the EZ-Cap recapper was -9 . We also found that the EZ-Cap recapper resolved some of the safety issues but in turn resulted in other technical and safety problems.

\section{DISCUSSION}

Overall, our nuclear medicine technologists preferred the Mayo recapper. The pros of this device outweighed its cons, as evidenced in the scores of our survey.

Use of the Mayo recapper has been implemented in our practice for about $8 \mathrm{y}$, and it has been determined that this device is more comfortable for our nuclear medicine technologists to use than the EZ-Cap recapper. This comfort level may be due to the fact that the Mayo recapper has been used more extensively in our practice than the EZ-Cap recapper.

The 2 holes in the Mayo recapper allow the use of butterfly needles and syringes. This feature makes the device perfect for 3-phase bone injections and for injections for pediatric patients, during which butterfly needles and syringes may need to be used simultaneously. The Mayo recapper is a stable device, and the nonslip feet help prevent its movement during use. Most importantly, no twisting motion is required to use this capping block, and the technologist can see and feel when the needle is recapped. These characteristics make the Mayo recapper simple to use.

The biggest drawback of the Mayo recapper is that 2 hands are needed to uncap a syringe. This fact alone may be the main reason why a needlestick can occur during its use.

The EZ-Cap recapper is a single-handed device. This feature presents a huge advantage over the Mayo recapper and can potentially lead to fewer needlesticks and less injury for technologists performing patient injections. The suction cup on the bottom of the device keeps the recapper stable during use and allows it to adhere to many different surfaces. Finally, the slanted angle of the EZ-Cap recapper allows easier capping and uncapping of a needle than does the Mayo recapper.

The EZ-Cap recapper was disliked for several technical reasons. The twisting action needed to uncap a needle is not optimal. This action made the device difficult and awkward to use. Technologists must twist their wrist to initiate grabbing of a needle cap by the device. This twisting action could possibly lead to difficulty in uncapping or capping a needle, to contamination, or to loss of the needle and needle cap before injection. Additionally, after twisting the wrist 
and the syringe to secure the needle cap, the technologist must manipulate an exposed needle before giving the injection to the patient. Manipulation of an exposed needle in this manner includes bringing the syringe from the capping block to the patient, holding the syringe while securing a vein, positioning the uncapped needle to puncture the vein properly, and moving the uncapped needle back to the capping block. This manipulation may result in needlesticks or contamination. The risk of pushing the plunger of the syringe when uncapping a needle is increased and may result in loss of radioactivity from the syringe before injection or contamination after the plunger is pushed. Finally, the EZ-Cap recapper has room for only 1 needle cap; this device is not practical to use when 2 needles are needed for a patient injection.

During the survey time for this project, there were no contamination issues resulting from use of the EZ-Cap recapper. However, there were several situations in which contamination could potentially have taken place during use of the EZ-Cap recapper. These situations resulted from the technical difficulties involving the EZ-Cap recapper discussed here.

These cons of the EZ-Cap recapper led to the Mayo recapper being chosen over the EZ-Cap recapper. The Mayo recapper is simple and easy to use and may be implemented in any hospital that performs patient injections.

\section{CONCLUSION}

We chose to continue using the Mayo recapper as our primary capping block. Because of a few safety reasons, the Mayo recapper needs to be improved. The EZ-Cap recapper resolves some of these problems but, in turn, leads to others.

We plan to continually monitor the safety and effectiveness of the Mayo recapper to prevent needlesticks. Further investigation may be needed to develop a capping block that improves on the cons of both the Mayo recapper and the EZ-Cap recapper. An ideal capping block should not only limit the potential for needlesticks but also be simple in its design and use.

\section{ACKNOWLEDGMENT}

Portions of this article were published in abstract form in J Nucl Med. 2007;48(suppl 2):466P.

\section{REFERENCES}

1. Lee JM, Bottleman MF, Xanthakos N, Nicklasson L. Needlestick injuries in the United States: epidemiologic, economic, and quality of life issues. AAOHN J. 2005;53:117-133.

2. Hung JC, Krause SJ, Schmit CL. Sensible approaches to avoid needle stick accidents in nuclear medicine. J Nucl Med Technol. 1999;27:290-293.

3. Hagerty J. Karen Daley, RN, MPH-On Needlestick Safety. Hoffman Estates, IL: Nursing Spectrum; 2004. Available at: http://www2.nursingspectrum.com/ articles/article.cfm?aid=18831. Accessed August 1, 2008. 